\title{
Avaliação de modelos de predição da energia metabolizável do milho para suínos
}

\author{
[Evaluating models to predict the metabolizable energy of maize for swine] \\ R.N. Pelizzeri ${ }^{1}$, P.C. Pozza ${ }^{2 *}$, N.T.E. Oliveira ${ }^{3}$, M.L. Somensi ${ }^{1}$, A.C. Furlan ${ }^{2}$, M.E. Neumann ${ }^{1}$ \\ ${ }^{1}$ Aluno - CCA-UNIOESTE - Marechal Cândido Rondon, PR \\ ${ }^{2}$ Universidade Estadual de Maringá - Maringá, PR \\ ${ }^{3}$ Centro de Ciências Agrárias - UNIOESTE - Marechal Cândido Rondon, PR
}

\begin{abstract}
RESUMO
Os objetivos propostos no presente trabalho foram a validação da predição de modelos de regressão linear de $1^{\circ}$ grau, dos valores estimados de energia metabolizável (EM) em função dos valores observados de EM do milho, obtidos em ensaios biológicos com suínos. Setenta e quatro registros de composição química e energética do milho foram obtidos na literatura e utilizados para estimar a EM de 41 modelos de predição em função da composição química. A significância dos parâmetros $\left(\beta_{0}\right.$ e $\left.\beta_{1}\right)$ da regressão foi avaliada pelo teste $t$ parcial, e a validação da predição dos modelos de $1^{\circ}$ grau foi obtida pela aceitação da hipótese de nulidade conjunta $\beta_{0}=0$ e $\beta_{1}=1$. Os modelos $\mathrm{EM}_{7}=1,099+0,740 \mathrm{~EB}-5,5 \mathrm{MM}-3,7 \mathrm{FDN} ; \mathrm{EM}_{9}$ $=16,13-9,5 \mathrm{FDN}+16 \mathrm{EE}+23 \mathrm{~PB} * \mathrm{FDN}-138 \mathrm{MM} * \mathrm{FDN}$ e $\mathrm{EM}_{13}=5,42-17,2 \mathrm{FDN}-19,4 \mathrm{MM}+0,709 \mathrm{~EB}$ são os mais adequados para estimar os valores de EM do milho e podem ser utilizados como ferramenta para formulação de rações para suínos.
\end{abstract}

Palavras-chave: composição química, equações lineares, milho, validação

\begin{abstract}
The proposed objective of this study was to validate the prediction of linear regression models of the first degree, the estimated values of metabolizable energy (ME) regarding the observed ME values of maize obtained in biological assays with swine. Seventy four records of chemical and energetic composition of maize were obtained from literature and used to estimate the ME of 41 prediction models depending on the chemical composition. The significance of the regression parameters $\left(\beta_{0}\right.$ and $\left.\beta_{1}\right)$ was evaluated by partial $t$ test and the prediction validation of first degree models was obtained by accepting the null hypothesis $\beta_{0}=0$ and $\beta_{1}=1$. The $M E_{7}=1.099+0.740 G E-5.5 \mathrm{MM}-3.7 \mathrm{NDF} ; \mathrm{ME}_{9}=16.13-9.5 \mathrm{NDF}+$ $16 E E+23 C P^{*} N D F-138 M M^{*} N D F$ and $M E_{13}=5.42-17.2 \mathrm{NDF}-19.4 M M+0.709 \mathrm{GE}$ models are the most adequate to estimate the metabolizable energy of maize and can be used as a tool to formulate diets for swine.
\end{abstract}

Keywords: chemical composition, corn, linear equations, validation

\section{INTRODUÇÃO}

Para se obter uma dieta que atenda às exigências nutricionais dos suínos, é necessário o conhecimento preciso dos valores de energia metabolizável dos alimentos utilizados na formulação de rações, e esses valores podem ser determinados por meio de métodos diretos e indiretos.

Recebido em 10 de março de 2011

Aceito em 5 de novembro de 2012

* Autor para correspondência (corresponding author)

E-mail: pcpozza@uem.br
Os métodos diretos requerem a realização de ensaios metabólicos em que se utilizam metodologias trabalhosas, demoradas e dispendiosas, o que dificulta sua utilização pela indústria suinícola. O método indireto consiste no uso de modelos matemáticos que predizem os valores energéticos dos alimentos.

As equações de predição podem ser baseadas na composição proximal dos alimentos, obtidas 
rotineiramente em laboratórios, sendo consideradas uma alternativa rápida, prática e econômica na avaliação nutricional dos alimentos (Ferreira et al., 1997; Zonta et al., 2004). Desta forma, as análises laboratoriais devem ser realizadas com critério, pois os resultados podem ser utilizados para se formularem as rações e para se estimarem os valores de energia metabolizável dos alimentos por meio de equações de predição.

Vários trabalhos (Noblet e Perez, 1993; Ferreira et al., 1997; Pozza et al., 2008) foram realizados com o intuito de ajustar modelos que estimem a energia metabolizável de ingredientes para suínos, entretanto não se conhecem adequadamente a aplicabilidade e a validade dos modelos em amostras diferentes.

A maioria dos modelos apresenta bom poder de predição para o conjunto de dados em que eles foram derivados (Oliveira e Warpechowski, 2009). No entanto, há poucos estudos que avaliam o comportamento de modelos de predição em um conjunto de dados independentes com o intuito de avaliar a precisão desses modelos.

As equações de predição dos valores de energia metabolizável dos alimentos utilizados em rações para suínos, uma vez validadas, podem ser utilizadas nas indústrias como instrumento para aumentar a precisão no processo de formulação de rações.

Entre os alimentos utilizados em rações para suínos, o milho contribui com o maior aporte de energia metabolizável. Porém, o conteúdo de nutrientes do milho, encontrado em tabelas de composição, apresenta variação na composição química em relação aos valores mensurados em laboratório, e entre os fatores que determinam tal variação pode-se citar a variedade cultivada (Sakomura e Silva, 1998). Essa variação na composição química do milho pode proporcionar diferentes valores de energia metabolizável em relação aos valores encontrados em tabelas de composição de alimentos, e o uso de equações apropriadas, que predizem com precisão os valores de energia metabolizável, pode ser uma alternativa viável.

Frente a tal situação, o presente trabalho foi realizado com o objetivo tanto de validar modelos de predição dos valores de energia metabolizável do milho para suínos, utilizando um conjunto de dados independentes obtidos na literatura nacional, quanto de selecionar os modelos validados de acordo com os maiores valores de probabilidade de significância.

\section{MATERIAL E MÉTODOS}

Informações sobre ensaios de metabolismo foram coletadas em pesquisas disponibilizadas na literatura científica nacional, com o objetivo de elaborar um banco de dados sobre composição química e valores de energia bruta (EB) e energia metabolizável (EM) do milho para suínos.

Os dados de composição química coletados foram: proteína bruta $(\mathrm{PB})$, extrato etéreo (EE), matéria mineral $(\mathrm{MM})$, cálcio $(\mathrm{Ca})$, fósforo $(\mathrm{P})$, fibra em detergente neutro (FDN), fibra em detergente ácido (FDA), fibra bruta (FB) e amido (amido). De posse da composição química, foi calculado o extrativo não nitrogenado (ENN), a matéria orgânica (MO) e a hemicelulose (Hemi).

Após a coleta das informações, foi realizada uma rigorosa e minuciosa triagem dos valores de composição química e dos valores energéticos, obtendo-se um total de 74 observações de composição química, energia bruta e energia metabolizável do milho para suínos, no período de 1985 a 2009. Os dados coletados dos ensaios de metabolismo foram convertidos para $100 \%$ de matéria seca para futura predição da EM.

Um total de 41 equações de predição dos valores de EM para suínos, em função da composição química, foi obtido na literatura e utilizado para gerar os valores de energia metabolizável estimada (EME).

As equações $\mathrm{EM}_{1}=4369-10,9 \mathrm{MM}+4,1 \mathrm{EE}-$ $6,5 \mathrm{FB}\left(\mathrm{R}^{2}=0,87\right) ; \mathrm{EM}_{2}=4,334-8,1 \mathrm{MM}+$ $4,1 \mathrm{EE}-3,7 \mathrm{FDN}\left(\mathrm{R}^{2}=0,91\right) ; \mathrm{EM}_{3}=4168-12,3$ $\mathrm{MM}+1,4 \mathrm{~PB}+4,1 \mathrm{EE}-6,1 \mathrm{FB}\left(\mathrm{R}^{2}=0,88\right) ; \mathrm{EM}_{4}=$ $4,194-9,2 \mathrm{MM}+1,0 \mathrm{~PB}+4,1 \mathrm{EE}-3,5 \mathrm{FDN}\left(\mathrm{R}^{2}=\right.$ $0,92) ; \mathrm{EM}_{5}=4,182-9,6 \mathrm{MM}+1,1 \mathrm{~PB}+4,1 \mathrm{EE}-$ 2,4 Hemi - 4,4FDA $\left(\mathrm{R}^{2}=0,93\right) ; \mathrm{EM}_{6}=1,255+$ $0,712 \mathrm{~EB}-8,5 \mathrm{MM}-6,6 \mathrm{FB}\left(\mathrm{R}^{2}=0,85\right)$ e $\mathrm{EM}_{7}=$ $1,099+0,740 \mathrm{~EB}-5,5 \mathrm{MM}-3,7 \mathrm{FDN}\left(\mathrm{R}^{2}=0,85\right)$ foram propostas por Noblet e Perez (1993). 
A equação $\mathrm{EM}_{8}=4,184,32-2,683 \mathrm{~PB}+1,723 \mathrm{EE}$ - 62,751P $\left(\mathrm{R}^{2}=0,19\right)$, proposta por Castilha (2007), foi ajustada especificamente para o uso na predição da EM do milho.

As equações $\mathrm{EM}_{9}=16,13-9,5 \mathrm{FDN}+16 \mathrm{EE}+$ $23 \mathrm{~PB} * \mathrm{FDN}-138 \mathrm{MM} * \mathrm{FDN}\left(\mathrm{R}^{2}=0,39\right) ; \mathrm{EM}_{10}=$ $17,50-15,3 \mathrm{FDN}+16 \mathrm{EE}+5,9 \mathrm{~PB}-34 \mathrm{MM}\left(\mathrm{R}^{2}=\right.$ $0,40) ; \mathrm{EM}_{11}=18,47-21 \mathrm{FDN}+16 \mathrm{EE}+$ $30 \mathrm{~PB} * \mathrm{FDN}-32 \mathrm{MM}\left(\mathrm{R}^{2}=0,40\right) ; \mathrm{EM}_{12}=10,33$ $8,5 \mathrm{FDN}+18 \mathrm{EE}+9,7 \mathrm{~PB}+6,7$ Amido $\left(\mathrm{R}^{2}=0,41\right)$ e $\mathrm{EM}_{13}=5,42-17,2 \mathrm{FDN}-19,4 \mathrm{MM}+0,709 \mathrm{~EB}$ $\left(\mathrm{R}^{2}=0,43\right)$ foram propostas por Morgan et al. (1987) para o uso na predição da EM de alimentos em geral.

As equações $\mathrm{EM}_{14}$ até $\mathrm{EM}_{37}$, propostas por Just et al. (1984), em que: $\mathrm{EM}_{14}=17498-49,6 \mathrm{FB}\left(\mathrm{R}^{2}=\right.$ $0,83) ; \mathrm{EM}_{15}=-13534-55,7 \mathrm{FB}+1,7 \mathrm{~EB}\left(\mathrm{R}^{2}=\right.$ $0,96) ; \mathrm{EM}_{16}=-39013+31,1 \mathrm{MO}-49,3 \mathrm{FB}+$ $1,5 \mathrm{~EB}\left(\mathrm{R}^{2}=0,96\right) ; \mathrm{EM}_{17}=-28114+25,1 \mathrm{MO}+$ $18,1 \mathrm{EE}-50,5 \mathrm{FB}+1,1 \mathrm{~EB}\left(\mathrm{R}^{2}=0,97\right) \mathrm{e}^{\mathrm{EM}_{18}}=-$ $35105+35,9 \mathrm{MO}+5,1 \mathrm{~PB}+21,2 \mathrm{EE}-47,1 \mathrm{FB}+$ $0,9 \mathrm{~EB}\left(\mathrm{R}^{2}=0,97\right)$, foram propostas para a predição da EM de cereais.

As equações $\mathrm{EM}_{19}=16482-35,7 \mathrm{FB}\left(\mathrm{R}^{2}=0,69\right)$; $\mathrm{EM}_{20}=15894+10,3 \mathrm{EE}-35,1 \mathrm{FB}\left(\mathrm{R}^{2}=0,74\right) ;$ $\mathrm{EM}_{21}=15240+3,3 \mathrm{~PB}+9,5 \mathrm{EE}-34,9 \mathrm{FB}$ $\left(\mathrm{R}^{2}=0,76\right) ; \mathrm{EM}_{22}=16662-37,5 \mathrm{FB}\left(\mathrm{R}^{2}=0,70\right)$; $\mathrm{EM}_{23}=9038-37,3 \mathrm{FB}+0,4 \mathrm{~EB}\left(\mathrm{R}^{2}=0,75\right) ; \mathrm{EM}_{24}$ $=15527-26,0 \mathrm{FB}\left(\mathrm{R}^{2}=0,42\right) ; \mathrm{EM}_{25}=-4037+$ $17 \mathrm{MO}-27,6 \mathrm{FB}\left(\mathrm{R}^{2}=0,73\right) ; \mathrm{EM}_{26}=-10878+$ $17 \mathrm{MO}-28,3 \mathrm{FB}+0,6 \mathrm{~EB}\left(\mathrm{R}^{2}=0,84\right) ; \mathrm{EM}_{27}=-$ $10883+17,5 \mathrm{MO}-19,1 \mathrm{FB}-4,5 \mathrm{FDN}+0,6 \mathrm{~EB}$ $\left(\mathrm{R}^{2}=0,85\right) ; \mathrm{EM}_{28}=16155-30,6 \mathrm{FB}\left(\mathrm{R}^{2}=0,76\right)$; $\mathrm{EM}_{29}=9141-31,2 \mathrm{FB}+0,4 \mathrm{~EB}\left(\mathrm{R}^{2}=0,79\right) ; \mathrm{EM}_{30}$ $=9305-19,7 \mathrm{FB}-5,8 \mathrm{FDN}+0,4 \mathrm{~EB}\left(\mathrm{R}^{2}=0,82\right)$; $\mathrm{EM}_{31}=15858-28,1 \mathrm{FB}\left(\mathrm{R}^{2}=0,46\right) ; \mathrm{EM}_{32}=-3068$ $+20,2 \mathrm{MO}-28,7 \mathrm{FB}\left(\mathrm{R}^{2}=0,7\right) ; \mathrm{EM}_{33}=-9270+$ $16,4 \mathrm{MO}-29,3 \mathrm{FB}+0,5 \mathrm{~EB}\left(\mathrm{R}^{2}=0,80\right) ; \mathrm{EM}_{34}=$ $15748-27,6 \mathrm{FB}\left(\mathrm{R}^{2}=0,46\right) ; \mathrm{EM}_{35}=-3001+$ $20 \mathrm{MO}-28,3 \mathrm{FB}\left(\mathrm{R}^{2}=0,70\right) ; \mathrm{EM}_{36}=-9222+$ $16,3 \mathrm{MO}-28,8 \mathrm{FB}+0,5 \mathrm{~EB}\left(\mathrm{R}^{2}=0,80\right)$ e $\mathrm{EM}_{37}=-$ $9086+16,5 \mathrm{MO}-21,7 \mathrm{FB}-3,4 \mathrm{FDN}+0,5 \mathrm{~EB}$ $\left(\mathrm{R}^{2}=0,81\right)$ foram propostas para o uso na predição de alimentos em geral, e as equações $E_{28}, E_{29}$ e $E_{30}$ foram propostas para a predição de EM de alimentos de origem vegetal.

As equações $\mathrm{EM}_{38}=16,81-0,031 \mathrm{FB}\left(\mathrm{R}^{2}=0,68\right)$ e $\mathrm{EM}_{39}=16,34-0,034 \mathrm{FB}+0,003 \mathrm{~PB}\left(\mathrm{R}^{2}=0,73\right)$ foram propostas por Lekule et al. (1990) para o uso na predição da EM de alimentos em geral.
A equação $\mathrm{EM}_{40}=3221,47+60,91 \mathrm{~PB}-$ $29,04 \mathrm{MM}\left(\mathrm{R}^{2}=0,86\right)$ foi proposta por Ferreira et al. (1997) para o uso na predição da EM de alimentos energéticos, e a equação $\mathrm{EM}_{41}=$ $3590,3819-32,3367 \mathrm{FB}-23,7758 \mathrm{FDN}+$ 12,4046 FDA $\left(\mathrm{R}^{2}=0,904\right)$ foi ajustada por Costa et al. (2008) para a predição da EM de alimentos concentrados energéticos. $\mathrm{O}$ sufixo $\mathrm{EM}_{\mathrm{x}}$ corresponde ao número das equações utilizadas.

Antes de se efetuar a predição dos valores estimados de EM em relação aos valores observados, procedeu-se à análise dos resíduos padronizados de Student (RStudent), a fim de se diagnosticarem observações influentes ou outliers que pudessem interferir nas estimativas dos parâmetros $\beta_{0}$ e $\beta_{1}$ da regressão, a partir dos valores da variável regressora (energia metabolizável observada), obtidos de valores observados de EM em ensaios biológicos. Os valores preditos $(\hat{Y})$ de cada modelo de $1^{\circ}$ grau, os valores reais do resíduo $\left(\varepsilon_{\mathrm{i}}\right)$ e os valores RStudent $\left(\mathrm{r}_{\mathrm{i}}\right)$ foram gerados por utilização de uma barra (/) após o comando MODEL e com inclusão das opções " $p$ " (predito) e "r" (resíduo studentizado) do procedimento REG (SAS, 1999).

O critério adotado para identificação de outliers foi baseado na curva de distribuição normal; ou seja, valores de RStudent maiores que dois desvios-padrão, em valor absoluto, foram considerados como influentes.

A predição de valores estimados de EM (EME), em função dos valores observados de EM (EMO), foi feita por meio de ajuste de modelos de regressão linear de $1^{\circ}$ grau, utilizando-se o método dos mínimos quadrados. A detecção da significância das estimativas dos parâmetros $\beta_{0} \mathrm{e}$ $\beta_{1}$ foi verificada pelo teste " $t$ " parcial, aplicado individualmente a cada parâmetro. A ausência de significância para o coeficiente angular do modelo proposto indicou a inexistência de relação linear entre EME e EMO, ou seja, os valores de EMO não explicaram a variação nos valores de EME.

No caso em que a predição do modelo de $1^{\circ}$ grau não apresentou significância $(\mathrm{P}>0,05)$ para $\mathrm{O}$ intercepto, este foi retirado do modelo, sendo ajustado o modelo $Y_{i}=\beta_{1} X_{i}+\varepsilon_{i}$. A análise foi reprocessada, e a hipótese de nulidade $\left(\mathrm{H}_{0}\right)$ testada foi a relacionada ao coeficiente angular 
$\left(\beta_{1}=0\right)$, contra a hipótese alternativa bilateral $\left(\mathrm{H}_{\mathrm{a}}\right.$ : $\left.\beta_{1} \neq 0\right)$.

A validação da predição dos modelos de $1^{\circ}$ grau, e em consequência a validação das equações encontradas na literatura como preditoras da relação linear de energia metabolizável a partir dos valores de composição química, foi feita a partir do ajuste de um modelo linear de $1^{\circ}$ grau, dos valores preditos $(\hat{Y})$ em função dos valores estimados (Y). A hipótese de nulidade testada foi a hipótese conjunta para os parâmetros da regressão linear, em que $\beta_{0}=0$ e $\beta_{1}=1$ versus $\mathrm{H}_{\mathrm{a}}: \beta_{0} \neq 0$ e $\beta_{1} \neq 1$.

A significância dos parâmetros da regressão foi verificada aplicando-se o teste F, ao nível de 5\% de significância, conforme a metodologia descrita por Montgomery et al. (2006). A não rejeição de $\mathrm{H}_{0}$ determinou a semelhança entre os valores preditos $(\hat{\mathrm{Y}})$ e estimados $(\mathrm{Y})$, indicando que os valores de resíduo $\left(\varepsilon_{\mathrm{i}}=\mathrm{Y}-\hat{\mathrm{Y}}\right)$ da análise de regressão foram semelhantes.

Como critérios de seleção das equações validadas, foram utilizados os maiores valores de probabilidade de significância $(P$-value $)$, obtidos por meio do teste $\mathrm{F}$ dos valores preditos $(\hat{\mathrm{Y}}) \mathrm{em}$ função dos valores estimados de energia metabolizável, além de terem sido selecionadas equações que apresentaram a estimativa do intercepto significativa.

\section{RESULTADOS E DISCUSSÃO}

Os valores médios obtidos para matéria seca (MS), PB, FDN, FDA, EE, MM, energia bruta (EB) e energia metabolizável (EM) (Tab. 1) encontraram-se próximos aos valores corrigidos para a matéria seca, propostos por Rostagno et al. (2005), no entanto o valor médio da FB não apresentou tal proximidade.

Dados apresentados por Brasil (1996) mostraram valores de $9,0 \%$ de PB e $3315 \mathrm{kcal} / \mathrm{kg}$ de EM, na matéria natural, que também foram próximos aos valores médios apresentados na Tab. 1, todavia os demais valores médios obtidos não corresponderam aos apresentados na literatura supracitada.
O banco de dados utilizado no presente estudo apresentou maior coerência com os valores médios propostos por Rostagno et al. (2005) em relação aos apresentados por Brasil (1996), mostrando que, embora a revisão de literatura tenha sido realizada com trabalhos publicados de 1985 a 2009, os valores médios de EM e a composição química refletiram os valores médios de variedades de milho mais atuais.

Os dados de MS (Tab. 1) se assemelharam aos obtidos por Oliveira e Warpechowski (2009), que trabalharam com um total de 76 amostras de milho, cujo valor médio foi de $87,0 \%$ de MS. Entretanto, a amplitude dos valores de MS (Tab. 1) não foi coerente com os observados pelos autores, que encontraram valores mínimo e máximo de 76,1 e $89,8 \%$, respectivamente.

Além da MS, observou-se ampla variação entre os valores mínimos e máximos de composição química e EM (Tab. 1). Entre todos os nutrientes avaliados, o conteúdo de FDA $(47,2 \%)$ do milho foi o que apresentou maior coeficiente de variação, seguido pelos teores de FB $(27,1 \%)$, FDN (24,9\%), EE $(24,4 \%)$ e MM $(18,1 \%)$. Oliveira e Warpechowski (2009) também encontraram a maior variabilidade para a FDA $(31,7 \%)$ de 76 amostras de milho. Essa variação na composição química pode ter ocorrido devido a diversos fatores, entre eles citam-se as causas genéticas, que contribuem para a variação do teor de extrato etéreo, carboidratos e aminoácidos, e as condições ambientais, que, ao atuarem severamente sobre a cultivar, podem diminuir o conteúdo de óleo e o de proteína bruta (Passos, 2004).

A variação nos valores da composição química demonstrou que a utilização de valores fixos, em matrizes de programas de formulação de rações, pode ocasionar o desbalanceamento da dieta. Essa variação proporcionou diferenças nos valores de EM, uma vez que esta se encontra relacionada com a composição química do alimento. No presente trabalho, observou-se uma variação de EM do milho de 3081 a $4407 \mathrm{kcal} / \mathrm{kg}$ de MS (Tab. 1), que justifica a utilização de técnicas mais precisas para se obterem tais valores, a exemplo das equações de predição dos valores energéticos. 
Tabela 1. Estatísticas descritivas para os teores de matéria seca, proteína bruta, matéria mineral, extrato etéreo, cálcio, fósforo, fibra em detergente neutro (FDN), fibra em detergente ácido (FDA), amido, fibra bruta, energia bruta (EB) e energia metabolizável (EM) de amostras de milho, expressas com base na matéria seca

\begin{tabular}{lcccccc}
\hline Nutriente $(\%)$ & $\mathrm{N}$ & Média & $\begin{array}{c}\text { Desvio- } \\
\text { padrão }\end{array}$ & Mínimo & Máximo & CV $(\%)$ \\
\hline Matéria seca & 67 & 87,88 & 0,98 & 85,76 & 90,54 & 1,12 \\
Proteína bruta & 74 & 9,89 & 0,82 & 8,46 & 13,33 & 8,29 \\
Matéria mineral & 32 & 1,55 & 0,28 & 1,18 & 2,29 & 18,06 \\
Extrato etéreo & 67 & 3,93 & 0,96 & 0,70 & 5,96 & 24,43 \\
Cálcio & 58 & 0,032 & 0,02 & 0,001 & 0,08 & 62,5 \\
Fósforo & 57 & 0,295 & 0,04 & 0,150 & 0,413 & 13,56 \\
FDN & 49 & 13,94 & 3,47 & 9,00 & 23,97 & 24,89 \\
FDA & 48 & 4,43 & 2,09 & 2,40 & 13,88 & 47,18 \\
Amido & 15 & 73,83 & 2,66 & 69,41 & 77,21 & 3,60 \\
Fibra bruta & 56 & 2,62 & 0,71 & 1,21 & 6,23 & 27,10 \\
EB $(\mathrm{kcal} / \mathrm{kg})$ & 68 & 4490 & 175,89 & 3394 & 4738 & 3,92 \\
EM (kcal/kg) & 74 & 3794 & 194,64 & 3081 & 4407 & 4,34 \\
\hline
\end{tabular}

Houve variação entre os valores médios de EM, tanto estimados como observados (Tab. 2). A variação entre os valores energéticos do milho deve ser considerada ao se formularem rações nas indústrias, em razão do melhor aproveitamento dos nutrientes do milho pelos suínos. A formulação de rações baseada no uso de modelos matemáticos preditores da energia metabolizável do milho constitui ferramenta importante para os nutricionistas, pois as variações nos valores energéticos são consideradas, aumentando-se a precisão no atendimento das exigências de EM de suínos.

Tabela 2. Valores médios, desvios-padrão, valores mínimos e máximos, coeficientes de variação (CV) e valores de energia metabolizável estimada (EME) e observada (EMO) nos ensaios de metabolismo por modelo de predição, expressos na matéria seca

\begin{tabular}{|c|c|c|c|c|c|c|}
\hline Equação & $\mathrm{N}$ & $\begin{array}{c}\text { Média } \\
(\mathrm{kcal} / \mathrm{kg})\end{array}$ & $\begin{array}{l}\text { Desvio- } \\
\text { padrão }\end{array}$ & $\begin{array}{l}\text { Mínimo } \\
\text { (kcal/kg) }\end{array}$ & $\begin{array}{l}\text { Máximo } \\
\text { (kcal/kg) }\end{array}$ & $\mathrm{CV}(\%)$ \\
\hline $\mathrm{EME}_{2}$ & 11 & 3928 & 134,68 & 3645 & 4060 & 3,43 \\
\hline $\mathrm{EMO}_{2}$ & 11 & 3828 & 36,35 & 3762 & 3865 & 0,95 \\
\hline $\mathrm{EME}_{4}$ & 11 & 3892 & 136,13 & 3603 & 4024 & 3,50 \\
\hline $\mathrm{EMO}_{4}$ & 11 & 3828 & 36,35 & 3762 & 3865 & 0,95 \\
\hline $\mathrm{EME}_{5}$ & 11 & 3938 & 142,56 & 3628 & 4060 & 3,62 \\
\hline $\mathrm{EMO}_{5}$ & 11 & 3828 & 36,35 & 3762 & 3865 & 0,95 \\
\hline $\mathrm{EME}_{7}$ & 10 & 3901 & 88,02 & 3762 & 3998 & 2,26 \\
\hline $\mathrm{EMO}_{7}$ & 10 & 3825 & 37,09 & 3762 & 3865 & 0,97 \\
\hline $\mathrm{EME}_{9}$ & 9 & 4021 & 25,33 & 3960 & 4050 & 0,63 \\
\hline $\mathrm{EMO}_{9}$ & 9 & 3837 & 32,13 & 3762 & 3865 & 0,84 \\
\hline $\mathrm{EME}_{10}$ & 11 & 3905 & 135,15 & 3627 & 4042 & 3,46 \\
\hline $\mathrm{EMO}_{10}$ & 11 & 3828 & 36,35 & 3762 & 3865 & 0,95 \\
\hline $\mathrm{EME}_{11}$ & 11 & 3928 & 144,98 & 3639 & 4080 & 3,69 \\
\hline $\mathrm{EMO}_{11}$ & 11 & 3828 & 36,35 & 3762 & 3865 & 0,95 \\
\hline $\mathrm{EME}_{13}$ & 10 & 3923 & 94,54 & 3751 & 4031 & 2,41 \\
\hline $\mathrm{EMO}_{13}$ & 10 & 3825 & 37,09 & 3762 & 3865 & 0,97 \\
\hline $\mathrm{EME}_{30}$ & 27 & 4147 & 51,80 & 4056 & 4244 & 1,25 \\
\hline $\mathrm{EMO}_{30}$ & 27 & 3811 & 47,55 & 3708 & 3868 & 1,25 \\
\hline
\end{tabular}


Os valores médios de EM estimados pelos modelos $\mathrm{EM}_{2}, \mathrm{EM}_{4}, \mathrm{EM}_{5}, \mathrm{EM}_{7}, \mathrm{EM}_{9}, \mathrm{EM}_{10}$, $\mathrm{EM}_{11}, \mathrm{EM}_{13}$ e $\mathrm{EM}_{30}$ foram numericamente maiores do que seus respectivos valores médios observados (Tab. 2). As diferenças entre valores estimados e observados foram de $100\left(\mathrm{EM}_{2}\right), 64$ $\left(\mathrm{EM}_{4}\right), 110\left(\mathrm{EM}_{5}\right), 76\left(\mathrm{EM}_{7}\right), 184\left(\mathrm{EM}_{9}\right), 77$ $\left(\mathrm{EM}_{10}\right), 100\left(\mathrm{EM}_{11}\right), 98\left(\mathrm{EM}_{13}\right)$ e $336 \mathrm{kcal} / \mathrm{kg}$ $\left(\mathrm{EM}_{30}\right)$. Para esse último modelo, a diferença foi de $8,82 \%$ a favor da energia metabolizável estimada. Com base nos valores médios de EM, observou-se que os modelos de predição encontrados na literatura apresentaram a tendência de superestimar os valores médios de EM, quando confrontados com os valores energéticos observados.

Dos 41 modelos avaliados, nove apresentaram predição satisfatória da EM do milho, pois foi aceita a hipótese $\mathrm{H}_{0}: \beta_{0}=0$ e $\beta_{1}=1(\mathrm{P}>0,05)$ para as equações $\mathrm{EM}_{2}, \mathrm{EM}_{4}, \mathrm{EM}_{5}$ e $\mathrm{EM}_{7}$ (Noblet e Perez, 1993) e para $\mathrm{EM}_{9}, \mathrm{EM}_{10}, \mathrm{EM}_{11}, \mathrm{EM}_{13} \mathrm{e}$ $\mathrm{EM}_{30}$ (Just et al., 1984), sugerindo que houve semelhança estatística entre valores estimados (Y) e preditos de EM (Ŷ). Os valores de coeficiente de determinação $\left(\mathrm{R}^{2}\right)$, para esses modelos, variaram de 0,63( $\left.\mathrm{EM}_{11}\right)$ a $0,79\left(\mathrm{EM}_{13}\right)$, indicando que a aderência das equações de regressão aos dados de energia metabolizável estimada (EME) foi de magnitude média $\left(\mathrm{R}^{2}=\right.$ $0,63)$ a elevada $\left(R^{2}=0,79\right)($ Tab. 3$)$.

Para 19 modelos de predição avaliados, não houve significância $(\mathrm{P}>0,05)$ do coeficiente angular $\left(\beta_{1}\right)$ pelo teste $\mathrm{t}$ parcial, o que sugere inexistência de relação linear entre EME e EMO. Outros 13 modelos $\left(\mathrm{EM}_{6}, \mathrm{EM}_{8,}, \mathrm{EM}_{15}, \mathrm{EM}_{17}, \mathrm{EM}_{18}\right.$, $\mathrm{EM}_{23}, \mathrm{EM}_{25}, \mathrm{EM}_{26}, \mathrm{EM}_{29}, \mathrm{EM}_{32}, \mathrm{EM}_{33}, \mathrm{EM}_{35} \mathrm{e}$ $\left.\mathrm{EM}_{36}\right)$ apresentaram significância $(\mathrm{P}<0,05)$ para os dois parâmetros da regressão, fato que indica a existência das equações de regressão, contudo a hipótese conjunta $\left(\mathrm{H}_{0}: \beta_{0}=0\right.$ e $\left.\beta_{1}=1\right)$ foi rejeitada, mostrando que os valores estimados diferiram estatisticamente $(\mathrm{P}<0,05)$ dos valores preditos de EM (Tab. 3).

Este número reduzido de equações consideradas efetivas em predizer os valores de EM pode ser justificado, pois, apesar do grande esforço para elaborar modelos de predição, nem toda tentativa de relacionar composição química e energia tem obtido sucesso quando testada com dados independentes (Sibbald, 1982), o que pode estar associado à variabilidade entre diferentes laboratórios, observada mesmo quando a mesma técnica analítica é utilizada (Cromwell et al., 1999).

Entre as equações propostas por Noblet e Perez (1993) e que foram efetivas em predizer os valores de EM, observou-se que a $\mathrm{EM}_{2}$ e EM foram compostas por três variáveis regressoras, enquanto a $E_{4}$ apresentou quatro e a $E_{5}$ apresentou cinco regressoras. As equações propostas por Morgan et al. (1987) apresentaram, no máximo, quatro variáveis de composição química.

A utilização de número reduzido de variáveis de composição química para predizer os valores de EM é de grande importância, pois, de acordo com Pozza et al. (2008), as equações compostas por até quatro variáveis de composição química requerem menor tempo, maior facilidade e maior economia na determinação, podendo ser utilizadas com maior facilidade. No mesmo sentido, Wiseman e Cole (1985) relataram que um grande interesse tem sido demonstrado pela utilização de equações de predição de ED e EM dos alimentos compostas por apenas uma variável ou uma combinação de variáveis de composição química.

As equações $\mathrm{EM}_{2}, \mathrm{EM}_{4}, \mathrm{EM}_{5}, \mathrm{EM}_{7}, \mathrm{EM}_{9}, \mathrm{EM}_{10}$, $\mathrm{EM}_{11}, \mathrm{EM}_{13}$ e $\mathrm{EM}_{30}$ foram compostas por variáveis regressoras cujas análises laboratoriais são mais rápidas. No entanto, Rostagno et al. (2005) fizeram menção à equação $\mathrm{EM}=$ $4,952 \mathrm{~PB}_{\mathrm{d}}+9,45 \mathrm{G}_{\mathrm{d}}+4,14\left(\mathrm{MO}_{\mathrm{d}}-\mathrm{PB}_{\mathrm{d}}-\mathrm{G}_{\mathrm{d}}\right)$ para estimar o valor de energia metabolizável de alimentos de origem vegetal e produtos lácteos, em que "d" refere-se à fração digestível dos nutrientes; ou seja, essa equação utiliza variáveis regressoras baseadas em nutrientes digestíveis, em oposição às equações avaliadas no presente trabalho.

Observou-se que, entre as regressoras efetivas em predizer os valores de EM do milho, ao menos uma foi representada pela fração fibrosa (FB, FDN, FDA ou hemicelulose). Segundo Noblet e Perez (1993), a FB pode reduzir a digestão de outros nutrientes da dieta aumentando a secreção endógena, tanto de proteína quanto de gordura, associada ao aumento da massa microbiana. Além disso, a fermentação digestiva da fibra produz ácidos graxos voláteis, cuja eficiência metabólica é menor. 
Tabela 3. Equações de regressão dos valores estimados de energia metabolizável (EME) em função dos valores observados de EM $(X)$, coeficientes de determinação $\left(R^{2}\right)$ e significância do teste $F$ para os modelos de predição

\begin{tabular}{|c|c|c|c|}
\hline Equação da literatura & Equação de regressão & $\mathrm{R}^{2}$ & $P$-value \\
\hline $\mathrm{EM}_{1}$ & $\hat{Y}=4217,68$ & - & - \\
\hline $\mathrm{EM}_{2}$ & $\hat{Y}=-7376,15+2,95 X$ & 0,64 & 0,1298 \\
\hline $\mathrm{EM}_{3}$ & $\hat{\mathrm{Y}}=4155,65$ & - & - \\
\hline $\mathrm{EM}_{4}$ & $\hat{Y}=-7531,23+2,98 X$ & 0,64 & 0,1295 \\
\hline $\mathrm{EM}_{5}$ & $\hat{Y}=-8056,66+3,13 X$ & 0,64 & 0,1327 \\
\hline $\mathrm{EM}_{6}$ & $\hat{Y}=3161,25+0,26 X$ & 0,60 & 0,0172 \\
\hline $\mathrm{EM}_{7}$ & $\hat{Y}=-4119+2,10 X$ & 0,78 & 0,3716 \\
\hline $\mathrm{EM}_{8}$ & $\hat{\mathrm{Y}}=3519,20+0,075 \mathrm{X}$ & 0,16 & 0,0001 \\
\hline $\mathrm{EM}_{9}$ & $\hat{Y}=1540,15+0,65 X$ & 0,67 & 0,2499 \\
\hline $\mathrm{EM}_{10}$ & $\hat{Y}=-7458,05+2,97 X$ & 0,64 & 0,1318 \\
\hline $\mathrm{EM}_{11}$ & $\hat{Y}=-8212,77+3,17 X$ & 0,63 & 0,1271 \\
\hline $\mathrm{EM}_{12}$ & $\hat{Y}=3758,60$ & - & - \\
\hline $\mathrm{EM}_{13}$ & $\hat{Y}=-4717,79+2,26 X$ & 0,79 & 0,3809 \\
\hline $\mathrm{EE}_{14}$ & $\hat{\mathrm{Y}}=3850,20$ & - & - \\
\hline $\mathrm{EM}_{15}$ & $\hat{Y}=2191,43+0,50 X$ & 0,12 & 0,0001 \\
\hline $\mathrm{EM}_{16}$ & $\hat{Y}=4445,35$ & - & - \\
\hline $\mathrm{EM}_{17}$ & $\hat{Y}=3031,27+0,26 X$ & 0,40 & 0,0011 \\
\hline $\mathrm{EM}_{18}$ & $\hat{Y}=3206,52+0,25 X$ & 0,43 & 0,0019 \\
\hline $\mathrm{EM}_{19}$ & $\hat{Y}=3699,76$ & - & - \\
\hline $\mathrm{EM}_{20}$ & $\hat{Y}=3660,6$ & - & - \\
\hline $\mathrm{EM}_{21}$ & $\hat{Y}=3576,4$ & - & - \\
\hline $\mathrm{EM}_{22}$ & $\hat{Y}=3730,9$ & - & - \\
\hline $\mathrm{EM}_{23}$ & $\hat{\mathrm{Y}}=2994,9+0,19 \mathrm{X}$ & 0,26 & 0,0001 \\
\hline $\mathrm{EM}_{24}$ & $\hat{Y}=3536,1$ & - & - \\
\hline $\mathrm{EM}_{25}$ & $\hat{\mathrm{Y}}=3401,9+0,09 \mathrm{X}$ & 0,23 & 0,0001 \\
\hline $\mathrm{EM}_{26}$ & $\hat{Y}=3317,9+0,16 X$ & 0,44 & 0,0013 \\
\hline $\mathrm{EM}_{27}$ & $\hat{Y}=3982,6$ & - & - \\
\hline $\mathrm{EM}_{28}$ & $\hat{Y}=3655,6$ & - & - \\
\hline $\mathrm{EM}_{29}$ & $\hat{Y}=3088,78+18 X$ & 0,18 & 0,0001 \\
\hline $\mathrm{EM}_{30}$ & $\hat{\mathrm{Y}}=1,09 \mathrm{X}$ & - & 0,9759 \\
\hline $\mathrm{EM}_{31}$ & $\hat{Y}=3601,2$ & - & - \\
\hline $\mathrm{EM}_{32}$ & $\hat{Y}=3482,3+0,09$ & 0,22 & 0,0001 \\
\hline $\mathrm{EM}_{33}$ & $\hat{Y}=3151,6+0,15 X$ & 0,51 & 0,0060 \\
\hline $\mathrm{EM}_{34}$ & $\hat{\mathrm{Y}}=3578,3$ & - & - \\
\hline $\mathrm{EM}_{35}$ & $\hat{Y}=3452,4+0,09 X$ & 0,23 & 0,0001 \\
\hline $\mathrm{EM}_{36}$ & $\hat{Y}=3142,1+0,15 X$ & 0,51 & 0,0064 \\
\hline $\mathrm{EM}_{37}$ & $\hat{Y}=3743,6$ & - & - \\
\hline $\mathrm{EM}_{38}$ & $\hat{\mathrm{Y}}=3809,4$ & - & - \\
\hline $\mathrm{EM}_{39}$ & $\hat{Y}=3746,89$ & - & - \\
\hline $\mathrm{EM}_{40}$ & $\hat{Y}=3771,97$ & - & - \\
\hline $\mathrm{EM}_{41}$ & $\hat{Y}=3254,41$ & - & - \\
\hline
\end{tabular}

No mesmo sentido, Costa et al. (2008) observaram que a FDN foi a variável de maior importância em um modelo de predição ajustado com a utilização da meta-análise, uma vez que explicou mais de $87 \%$ da variação observada da EM $\left(R^{2}\right.$ parcial $\left.=0,8737\right)$. Os autores relataram ainda que a FDA e a FB não devem ser desconsideradas nas análises químicas, pois ambas foram importantes na explicação da variação energética dos alimentos concentrados energéticos.

As equações $\mathrm{EM}_{2}, \mathrm{EM}_{4}, \mathrm{EM}_{5}, \mathrm{EM}_{9} \mathrm{EM}_{10}$ e $\mathrm{EM}_{11}$ apresentaram a $\mathrm{MM}$ e o $\mathrm{EE}$ como variáveis independentes no modelo, mostrando-se como boas preditoras da energia metabolizável do 
milho. De acordo com Morgan et al. (1987), a MM apresentou efeito negativo para a EM, pois atuou como diluente da energia bruta, reduzindo o conteúdo de matéria orgânica dos alimentos, enquanto o EE apresentou efeito positivo. Além disso, as equações $\mathrm{EM}_{7}$ e $\mathrm{EM}_{13}$ têm a $\mathrm{MM}$ como variável independente.

A equação $\mathrm{EM}_{8}=4184,32-26,83 \mathrm{~PB}+17,23 \mathrm{EE}$ - 627,51P não apresentou uma boa confiabilidade em predizer os valores de EM do milho para suínos $(\mathrm{P}<0,05)$. Da mesma forma, Castilha et al. (2011), ao trabalharem com validação de modelos de predição de EM, inferiram que essa equação não apresentou uma boa capacidade em predizer os valores de EM do milho.

$\mathrm{Na}$ análise dos resíduos relacionados aos modelos de predição de $1^{\circ}$ grau de EME em função de valores de EMO do milho, o critério adotado para eliminar observações discrepantes de se utilizar valores de resíduo studentizado $\left(\mathrm{r}_{\mathrm{i}}\right)$ menores ou iguais a 2,00 desvios-padrão possibilitou validar modelos com valor absoluto de resíduo $\left(\varepsilon_{\mathrm{i}}\right)$ de até $135,90 \mathrm{kcal} / \mathrm{kg}\left(\mathrm{EM}_{11}\right)$, com
95\% de confiança. Para tais equações, houve equilíbrio no número de pontos com $\varepsilon_{\mathrm{i}}$ positivo $\left(\varepsilon_{\mathrm{i}}^{+}\right)$e $\varepsilon_{\mathrm{i}}$ negativo $\left(\varepsilon_{\mathrm{i}}^{-}\right)$(Tab. 4). O uso desse critério eliminou as observações discrepantes, atendendo à normalidade dos resíduos, um dos pressupostos para realização da análise de regressão linear.

A utilização do resíduo studentizado $\left(\mathrm{r}_{\mathrm{i}}\right)$ tornouse interessante porque, em sua estrutura matemática, há a ponderação para o desviopadrão de cada resíduo, sendo este diretamente relacionado ao quadrado médio residual da análise de variância da regressão. Desse modo, cada modelo de predição apresentou valores diferenciados de desvio-padrão e $r_{i}$. Foi constatado também que a observação com maior valor de resíduo $\left(\varepsilon_{\mathrm{i}}\right)$ pode não apresentar maior valor de $r_{i}$ por causa da ponderação pelo desviopadrão. Esse fato foi observado para as equações $\mathrm{EM}_{2}, \mathrm{EM}_{4}$ e $\mathrm{EM}_{9}$, que apresentaram o valor de $\varepsilon_{\mathrm{i}}$ com sinal positivo maior do que o respectivo valor de $\varepsilon_{\mathrm{i}}$ com sinal negativo, mas os valores absolutos de $r_{i}$ com sinal negativo foram maiores do que os respectivos valores de $r_{i}$ com sinal positivo (Tab. 4).

Tabela 4. Maiores e menores valores de resíduo $\left(\varepsilon_{\mathrm{i}}\right)$ e de resíduo studentizado $\left(\mathrm{r}_{\mathrm{i}}\right)$ e quantidade de pontos com resíduo positivo $\left(\varepsilon_{\mathrm{i}}^{+}\right)$e negativo $\left(\varepsilon_{\mathrm{i}}^{-}\right)$para os modelos de predição validados

\begin{tabular}{|c|c|c|c|c|c|c|}
\hline Equação & $\begin{array}{c}\text { Maior valor } \\
\text { de } \varepsilon_{\mathrm{i}}^{+}\end{array}$ & $\begin{array}{c}\text { Menor valor } \\
\text { de } \varepsilon_{\mathrm{i}}^{-}\end{array}$ & $\begin{array}{c}\text { Maior valor } \\
\text { de } r_{i}^{+}\end{array}$ & $\begin{array}{c}\text { Menor valor } \\
\text { de } r_{i}^{-}\end{array}$ & $\begin{array}{c}\text { Número de } \\
\text { pontos com } \\
\varepsilon_{\mathrm{i}}^{+}\end{array}$ & $\begin{array}{c}\text { Número de } \\
\text { pontos com } \\
\varepsilon_{\mathrm{i}}^{-} \\
\end{array}$ \\
\hline $\mathrm{EM}_{2}$ & 119,43 & $-114,17$ & 1,46 & $-1,63$ & 6 & 5 \\
\hline $\mathrm{EM}_{4}$ & 120,51 & $-117,35$ & 1,46 & $-1,67$ & 6 & 5 \\
\hline $\mathrm{EM}_{5}$ & 129,70 & $-129,93$ & 1,51 & $-1,77$ & 4 & 7 \\
\hline $\mathrm{EM}_{7}$ & 48,88 & $-66,09$ & 1,47 & $-1,60$ & 6 & 4 \\
\hline $\mathrm{EM}_{9}$ & 21,83 & $-13,57$ & 1,51 & $-1,77$ & 3 & 6 \\
\hline $\mathrm{EM}_{10}$ & 118,92 & $-119,14$ & 1,45 & $-1,53$ & 6 & 5 \\
\hline $\mathrm{EM}_{11}$ & 128,26 & $-135,90$ & 1,45 & $-1,59$ & 6 & 5 \\
\hline $\mathrm{EM}_{13}$ & 48,97 & $-76,88$ & 1,32 & $-1,75$ & 6 & 4 \\
\hline $\mathrm{EM}_{30}$ & 46,40 & $-50,29$ & 1,88 & $-2,03$ & 14 & 13 \\
\hline
\end{tabular}

Entre as equações validadas, os modelos $\mathrm{EM}_{7}$, $\mathrm{EM}_{9}, \mathrm{EM}_{13}$ e $\mathrm{EM}_{30}$ apresentaram os maiores valores de significância de $P$-value (Tab. 3), indicando que esses modelos apresentaram, de modo geral, erros com menores magnitudes, ou seja, maior semelhança numérica entre os pares de valores preditos e estimados. Entretanto, o modelo $\mathrm{EM}_{30}$ não apresentou intercepto significativo, podendo-se inferir que os modelos $\mathrm{EM}_{7}, \mathrm{EM}_{9}$ e $\mathrm{EM}_{13}$ foram os mais adequados para a predição dos valores de energia metabolizável do milho para suínos, que também apresentaram os menores valores absolutos de resíduos ordinários (Tab. 4). 


\section{CONCLUSÕES}

Os modelos $\mathrm{EM}_{7}=1,099+0,740 \mathrm{~EB}-5,5 \mathrm{MM}-$ $3,7 \mathrm{FDN} ; \mathrm{EM}_{9}=16,13-9,5 \mathrm{FDN}+16 \mathrm{EE}+$ $23 \mathrm{~PB} * \mathrm{FDN}-138 \mathrm{MM} * \mathrm{FDN}$ e $\mathrm{EM}_{13}=5,42-$ $17,2 \mathrm{FDN}-19,4 \mathrm{MM}+0,709 \mathrm{~EB}$ são os mais adequados para estimar os valores de EM do milho e podem ser utilizados como ferramenta para formulação de rações para suínos.

\section{REFERÊNCIAS}

BRASIL. Ministério da Agricultura, do Abastecimento e da Reforma Agrária. Normas e padrões de nutrição e alimentação animal: revisão. Curitiba: Maara, 1996, $145 \mathrm{p}$.

CASTILHA, L.D. Uso de modelos para predizer os valores energéticos de milho para suínos. 2007. 31f. Trabalho de Conclusão de Curso (Graduação em Zootecnia) - UNIOESTE, Universidade Estadual do Oeste do Paraná, Marechal Cândido Rondon, PR.

CASTILHA, L.D.; POZZA, P.C.; KLOSOWSKI, E.S. et al. Modelos matemáticos para predição dos valores energéticos do milho para suínos. Rev. Bras. Saúde e Prod. Anim., v.12, p.456-467, 2011.

COSTA, A.C.; LIMA, R.R.; FREITAS, R.T.F. Equação de predição dos valores de energia metabolizável aparente de alimentos concentrados energéticos para suínos, utilizando o princípio da meta-análise. In: REUNIÃO ANUAL DA SOCIEDADE BRASILEIRA DE ZOOTECNIA, 45., 2008, Lavras, MG. Anais... Lavras, MG, 2008. CDROM.

CROMWELL, G.L.; CALVERT, C.C.; CLINE, T.R. et al. Variability among sources and laboratories in nutrient analyses of corn and soybean meal. J. Anim. Sci., v.77, p.3262-3273, 1999.

FERREIRA, E.R.A.; FIALHO, E.T.; TEIXEIRA, A.S. et al. Avaliação da composição e determinação de valores energéticos e equação de predição de alguns alimentos para suínos. Rev. Bras. Zootec., v.26, p.514523, 1997.

JUST, A.; JORGENSEN, H.; FERNANDEZ, J.A. Prediction of metabolizable energy for pigs on the basis of crude nutrients in the feeds. Livest. Prod. Sci., v.11, p.105-128, 1984.

LEKULE, F.P.; JORGENSEN, J.P.; JUST, A. Nutritive value of some tropical feedstuffs for pigs: Chemical composition, digestibility and metabolizable energy content. Anim. Feed. Sci. Technol., v.28, p.91$101,1990$.
MONTGOMERY, D.C.; PECK, E.A.; VINING, G.G. Introduction to Linear Regression Analysis. 4th ed. New Jersey: John Wile \& Sons, Inc., 2006. p.612.

MORGAN, C.A.; WHITTEMORE, C.T.; PHILLIPS, P. et al. The prediction of the energy value of compounded pig foods from chemical analisys. Anim. Feed Sci. Technol., v.17, p.81-107, 1987.

NOBLET, J.; PEREZ, J.M. Prediction of digestibility of nutrients and energy values of pig diets from chemical analysis. J. Anim. Sci., v.71, p.3389-3398, 1993.

OLIVEIRA, V.; WARPECHOWSKI, M. Avaliação de modelos para predição da energia metabolizável do milho para aves. Cienc. Rural, v.39, p.1514-1520, 2009.

PASSOS, A.A.A. Variabilidade da composição nutricional do milho e seus efeitos no custo de rações para suínos. 2004. 46f. Dissertação (Mestrado em Zootecnia) - Faculdade de Agronomia Eliseu Maciel, Universidade Federal de Pelotas, Pelotas.

POZZA, P.C.; GOMES, P.C.; DONZELE, J.L. et al. Composição química, digestibilidade e predição dos valores energéticos da farinha de carne e ossos para suínos. Rev. Acta. Sci. Anim. Sci., v.30, p.33-40, 2008.

ROSTAGNO, H.S.; ALBINO, L.F.T.; DONZELE, J.L. et al. Tabelas brasileiras para aves e suínos: composição de alimentos e exigências nutricionais. 2. ed. Viçosa, MG: Universidade Federal de Viçosa, 2005. 186p.

SAKOMURA, N.K.; SILVA, R. Conceitos inovadores aplicáveis à nutrição de não ruminantes. Cad. Tec. Esc. Vet., v.22, p.125-146, 1998.

SAS INSTITUTE, SAS/STAT users guide. Version 6.12. Cary, N.C. 1999.

SIBBALD, I.R. Measurement of bioavailable energy in poultry feedingstuffs: a review. Can. J. Anim. Sci., v.62, p.983-1048, 1982.

ZONTA, M.C.M.; RODRIGUES, P.B.; ZONTA, A. et al. Energia metabolizável de ingredientes proteicos determinada pelo método de coleta e por equações de predição. Cienc. Agrotec., v.28, p.1400-1407, 2004.

WISEMAN, J.; COLE, D.J.A. Predicting the energy content of pig feeds. In: COLE, D.J.A.; HARESIGN, W. (Ed.) Recent developments in pig nutrient. London: Butterworth, 1985. p.59-70. 